\title{
Design of an Online Charging System to Support IMS-Based Inter-domain Composite Services
}

\author{
M. van Le ${ }^{1}$, G.B. Huitema ${ }^{2}$, F.J. Rumph ${ }^{3}$, L.J.M. Nieuwenhuis ${ }^{1}$, \\ and B.J.F. van Beijnum ${ }^{4}$ \\ ${ }^{1}$ Faculty of Information Systems and Change Management, University of Twente, \\ The Netherlands \\ ${ }^{2}$ Faculty of Economics and Business, University of Groningen, The Netherlands \\ ${ }^{3}$ TNO, The Netherlands \\ ${ }^{4}$ Faculty of Electrical Engineering, Mathematics and Computer Science, \\ University of Twente, The Netherlands \\ van-minh.le@atosorigin.com, g.b.huitema@rug.nl, \\ frens_jan.rumph@tno.nl, l.j.m.nieuwenhuis@utwente.nl, \\ beijnum@ewi.utwente.nl
}

\begin{abstract}
For service providers online charging of composite services is necessary in order to manage financial risks of service delivery in multi-domain environments. At service level, inter-domain composite services consist of one or more service components, e.g. access service, IMS communication service or content service, delivered by different service providers. The 3rd Generation Partnership Project (3GPP) has developed a framework for off-line and online charging of IMS-based services. However, the current Online Charging System (OCS) specified by 3GPPP does not support an online charging function for composite services. The contribution of this work lies in the design of an online charging system that addresses the required charging functionalities based on the IMS online charging architecture. The design consists of a service composition information model guided by the NGOSS and SID concepts of the TeleManagement Forum, a set of functional charging system components and their interactions at their interfaces.
\end{abstract}

Keywords: Online charging, IP Multimedia Subsystem (IMS), inter-domain composite services.

\section{Introduction}

Today, customers consume a broad variety of multimedia services. The growth of data-centric mobile devices and mobile broadband network capacity is pushing multimedia services into the mainstream. Most of these multimedia services are composite services. A composite service is composed of many service components provided by third party providers to the service provider.

In general there are off-line and online charging mechanisms. Here off-line charging means that the charging for service usage occurs after a service event or service session has occurred (i.e. postpaid). On the other hand online charging means that the 
charging occurs during a service session usage or right after a service event has occurred. We note that the notion of online charging may refer to prepaid as well as postpaid. In the latter case online charging will allow consumers to set charge limits when desired (e.g. roaming in foreign countries).

Now, the delivery of composite services imposes a huge complexity on online charging mechanisms, especially when dealing with dynamic online charging schemes. That is, when the charge of an individual service component depends on the existence of other components during a service session. For example, see Figure 1, a TVoD (TV-on-demand) session may be combined with an advertising service component. During the service session, the customer, here Jane, may decide to remove the advertising component, which results in an immediate increase of the TVoD service charge.

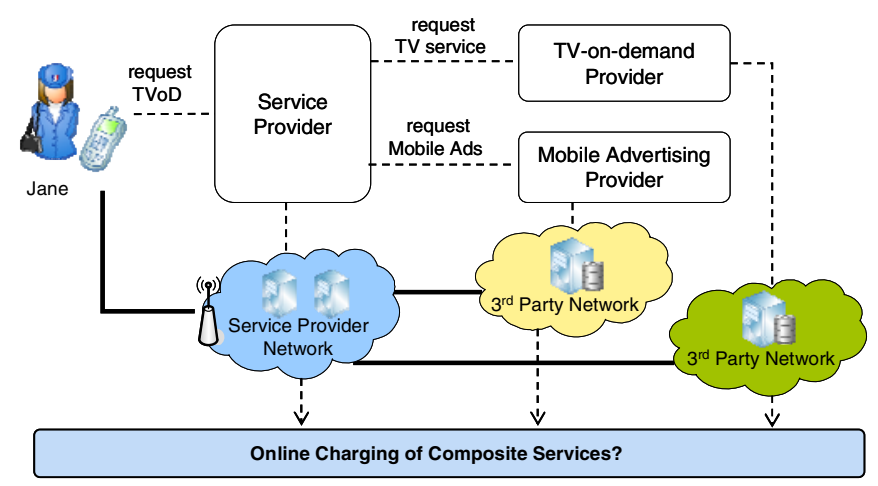

Fig. 1. Inter-domain service scenario depicting functional relationships (dash lines) and the physical paths of the delivered data (solid lines). The arrows and the blue box denote the problem domain of online charging across multiple administrative domains.

In order to deal with online charging of composite services based on dynamic pricing, new online charging architectures are needed. With the uptake of more valuable, complex services most customers of composite multimedia services want to obtain (near) real-time charging and billing information to manage their expenses during usage. Furthermore, also service providers need (near) real-time management information in order to manage their financial risks. However, today's charging systems are not capable of dealing with requirements related to composite services. In particular, the 3rd Generation Partnership Project (3GPP) has developed the IP Multimedia Sub-system (IMS) [1] to support multimedia services but their framework for current Online Charging Systems (OCS) do not support online charging functions for interdomain composite services.

The contribution of this work lies in the design of an online charging system to support IMS-based inter-domain composite services. The design uses the IMS online charging architecture as a basis to develop a refined online charging system that addresses the required charging functionalities. The design is also based on the New Generation Operations Systems and Software (NGOSS), in particular the Information Framework (SID), concepts of the TeleManagement Forum [9]. 
The remainder of this paper is organized as follow: Section 2 provides a brief overview of related work. Section 3 presents the problem domain of online charging of composite services. Section 4, 5 and 6 proposes a design of an online charging system solving the stated problem, by respectively defining a service composition information model, defining the functional charging system components and by defining the interactions at their interfaces. Finally, Section 7 presents the conclusion together with some future research directions.

\section{Related Work}

In this section related work with respect to online charging of composite services is described. The $3 \mathrm{GPP}$ has proposed two reference charging models for service scenarios [2], namely: an off-line and an online charging model. The latter charging model covers near real-time charging issues such as charging authorization, credit control during service sessions. However, it lacks a service composition model to deal with charging of composite services. For instance, a video conference can fall back from video plus voice to only voice due to some network problem. This introduces a change in charging of the voice component for the rest of the conversation. In this kind of situation, it is necessary for a charging system to keep track of the service composition information to adapt charging accordingly.

The Akogrimo project [3] has proposed a charging architecture called A4C (Authentication, Authorization, Accounting, Auditing, and Charging). A4C consists of a number of basic system components based on the IETF's AAA architecture [4]. This architecture offers charging solutions for mobile grid services in a multi-provider service environment and is designed to support charging of inter-domain composite services. However, the architecture lacks a specification of composite services, and real-time service charging and billing have not received detailed attention.

Koutsopoulou [5] has proposed an architecture to support billing processes which also accounts for inter-domain billing. In this architecture, the authentication, authorization and event-based billing in roaming service scenarios is well addressed. However, the charging of composite services has not been considered in detail.

$\mathrm{Xu}$ and Jennings [13] have proposed a framework for automated generation of charging schemes for composite IMS services. This framework provides a comprehensive approach to ease the modification of charging schemes. Nonetheless, it does not address the issues of online charging for composite IMS services.

Our approach contributes to the above related work in a number of ways. We propose a service composition information model and a charging system that deals with online charging of inter-domain composite services. To this extent, we propose a concrete refinement of the Online Charging System (OCS) that has been specified by the 3GPP thus far.

\section{Problem Domain of Online Charging of Composite Services}

This section deals with the focus of our research: the setup of an online charging system for inter-domain composite services. Depending on the implementation strategy online charging may involve different distributed Online Charging Systems (OCS). 
However, we abstract from a concrete distribution and focus on one single OCS. We assume that the composite services will be delivered across several administrative domains and across several delivery platforms. Hence, a combination of web services and IMS services based on SOA (Service Oriented Architecture) [6] is considered.

In order to master the complexity of such a service delivery, the concept of service broker as advocated in [6,7] is used. There are different possible configurations of service brokers in an actual deployment. Here we consider two types of service broker: 1. An IMS Service Broker within the IMS domain and 2. An Inter-domain Service Broker within the Web-Services domain. Here, the Inter-domain Service Broker is leading and is responsible for the end-to-end service composition for the user.

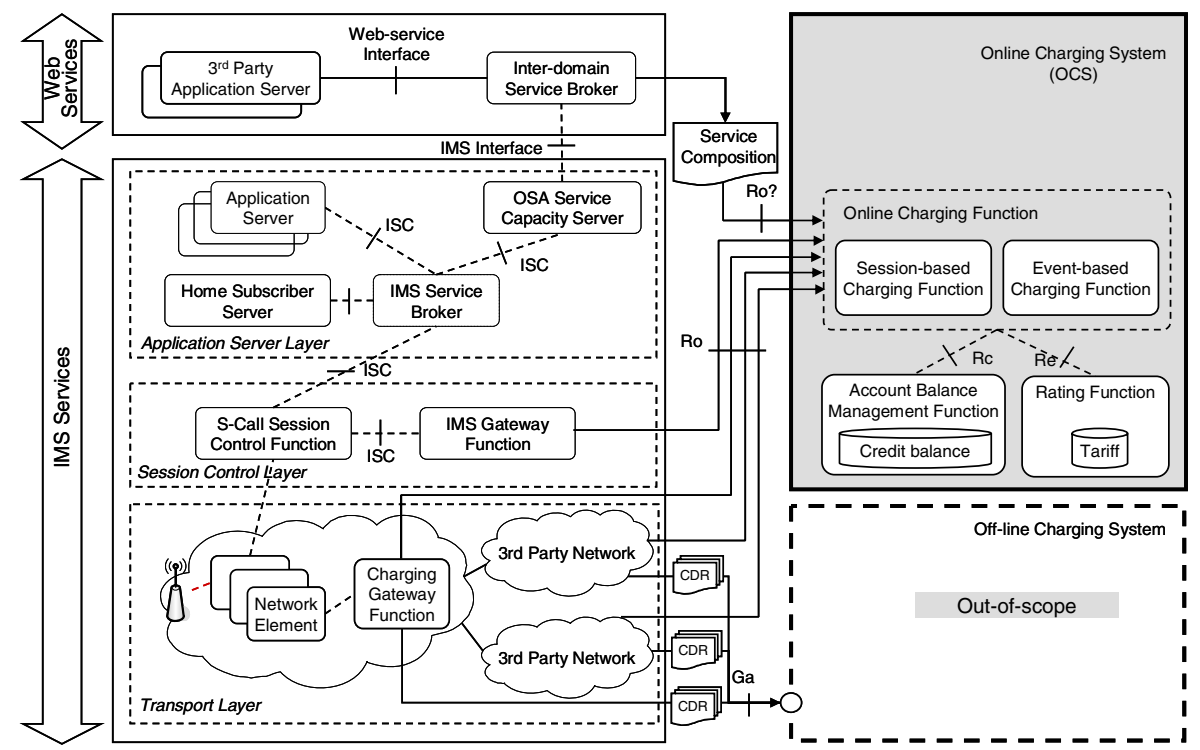

Fig. 2. Provisioning and Charging of Composite Services in an Inter-domain Environment

Figure 2 above illustrates a possible model for provisioning and charging of composite services in a multi-domain environment. A composite service is delivered across different administrative domains: the service provider domain, which is supported by IMS core infrastructure, and the third party domains, which are not necessarily supported by an IMS but some different network infrastructures. The service composition and orchestration occurs at the web-service level through the Inter-domain Service Broker. This implies that the Inter-domain Service Broker has the knowledge of the service composition and needs to communicate this information with the OCS for online charging purposes. Note that here off-line charging is out-of-scope.

The 3GPP framework describes online charging for both events and sessions. However, there exists no standard model yet for service composition within 3GPP specifications. Although an IMS service may consist of different service components (e.g. VoIP over IP-access), current online charging systems do not correlate charges between service components. As a result, when a service component is added or removed from an incurred composite service session, adjustment of online charging due 
to possible tariff changes (e.g. zero rate bearer usage when a VoIP session is active) cannot be handled. Since the composition of a composite service may change at run time, online charging needs to adapt to this dynamic behavior as well. This implies that the OCS needs to have knowledge about how the ongoing service composition is built up and the corresponding tariff of an individual service component. Furthermore, charging policies need to be enforced appropriately according to some pre-defined service level agreement between the customer and the service provider.

Hence we come up to the following research questions regarding online charging of inter-domain composite services:

1. What is the service composition information model used by the OCS?

2. What are the functional components embodied within the OCS?

3. How do these functional components interact at their interfaces?

The above questions will be addressed respectively in the sections 4, 5 and 6 below.

\section{A Service Composition Model}

This section presents a service composition information model for online charging of inter-domain composite services.

According to the Service Delivery Framework of the TeleManagement Forum (TMForum) [8], there are three steps to arrive at the eventual service delivery, namely: product design, service creation and service execution. During the product design phase, a service designer from the service provider domain can look up available service components in a catalog and chooses the necessary service components to form a composite service. During the creation phase, the designed composite service is tested throughout. When a composite is accepted, a meta information model of the composite service is created and stored in a composite service catalog. In the last phase, whenever a user requests a composite service, an instance to the corresponding meta information model is generated. Figure 3 depicts when a meta service composition model comes into existence.

In order to compose a composite service, the service provider mostly needs external services from third party providers. A service composition model therefore must express the relationship between the constituent service components. The TMForum

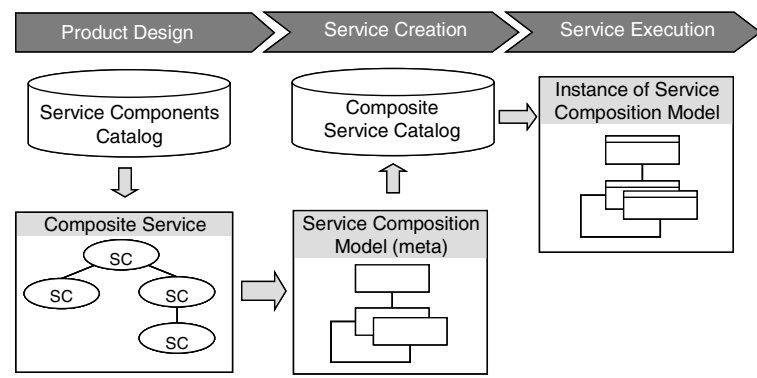

Fig. 3. Service Composition Information in the context of Service Delivery Framework of TMForum 
also has been working on the SID (Information Framework) model [9], which provides guidelines for the modeling of information/data for the purpose of product design, service construct and service provisioning. Currently, the SID model is widely accepted as standard in the industry. Among many aspects, the SID model addresses the basic entities: product, service, end-user-facing service and provider-facing service and their relationships.

The service composition information model contains necessary detail information to ensure the correlation of service components and their corresponding charge. Based on SID the following pieces of information are crucial:

- serviceID - a unique identifier of a provided composite service or a service component.

- interOperatorID - a unique identifier of a service provider or a third party provider.

- chargingKey - an identifier used by the OCS to determine the tariff of a composite service or a service component.

The combination of an interOperatorID, the corresponding serviceID and the chargingKey allows for an appropriate credit authorization request at the OCS. For more details on the service composition information model see our previous work in $[10,11]$.

\section{The Proposed Online Charging System}

This section describes the functional components embodied within the proposed Online Charging System (OCS). It presents a description of OCS and their interactions at the interfaces.

\subsection{Intertwinement of Service Life Cycle and Charging Life Cycle}

The rationale of the processes for the online charging of services is to intertwine the service life cycle and the charging life cycle as we have proposed in [12]. The intertwining of these two different life cycles is described by the process given in Figure 4.

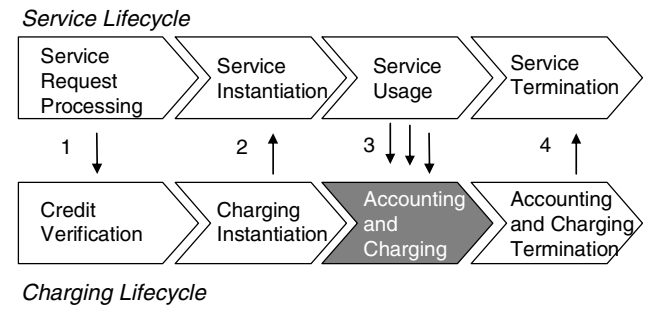

Fig. 4. Intertwinement of Service Life Cycle and Charging Life Cycle 
In short, the use of a service comprises the following phases:

1. Service Request Processing - In this phase, a request initiated by the end-user is processed.

2. Service Instantiation - In this phase, the requested service is orchestrated (i.e. composed) and set-up for use.

3. Service Usage - In this phase the service is actually provided for use by the service provider to the end-user.

4. Service Termination - In this phase the service use is terminated, this is either initiated by the end-user or by the service provider.

For the online charging of this service usage, the following phases are defined:

1. Credit Verification - This process validates if the Subscriber's credit conforms to the requirements allowing the service request to be granted.

2. Charging Instantiation - This process prepares the charging of the orchestrated service.

3. Accounting and Charging - During this phase usage events (i.e. usage records) are received by the OCS and processed as to determine in (near) real-time the charge of the service.

4. Accounting and Charging Termination - In this phase, the accounting and charging is terminated (gracefully).

For more details on the credit control mechanisms and the interactions with the Interdomain Service Broker see [11].

\subsection{Functional Components of the Online Charging System}

In this sub-section the refinement of the OCS is discussed. The OCS consists of the following functional components:

- Mediation - The Mediation acts as a gateway with the capability to receive and to route incoming messages from provisioning systems to the Charge Aggregator. As the Charge Aggregator is a computation-intensive system component, the Mediator can take care of the load balancing by distributing the charging process over multiple Charging Aggregator instances. The Mediation component has a port that connects to "outward" interfaces to provisioning systems.

- Charge Aggregator - The Charge Aggregator is mainly responsible for credit verification. It has knowledge of service composition information to conduct the accumulation of charge reservations (or claims) that belong to a particular service session. Unlike conventional post-paid billing where charge records are produced by rating engines, here charge records are produced by the Charge Aggregator. The rationale behind this design choice is related to the assigned intelligence of the Charge Aggregator to keep track of all states of all charging sessions and to aggregate the charges of the involved components. Hence, this information is used to generate the charge records.

- Balance Manager - The Balance Manager manages and updates the credit balances of subscribers. When a subscriber credit balance reaches a certain predefined threshold, the Balance Manager informs the Charge Aggregator about 
the balance status so that appropriate action can be taken by the provisioning system. For instance, the provisioning system may decide to proceed with the service provisioning based on the good history of the subscriber in question or the provisioning system may decide to immediately terminate the ongoing service provisioning.

- Rating Engine - The Rating Engine conducts the calculations for charge reservation for the individual service components based on the incoming charge requests. The Rating Engine retrieves the tariff and discount information (i.e. user specific charging profile) from the Tariff \& Discount Database for a specific End-user and calculates the costs for different service components involved. The Rating Engine retains the tariff and discount information for the entire Charging Life Cycle.

- Balance Database - The Balance Database stores the credit balance information of subscribers. When a charging session starts, the Balance Manager retrieves the current credit balance status of a specific End-user and his associated Subscriber from the Balance Database. The state of the credit balance changes during a charging session and the Balance Manager maintains this state. When the charging session terminates, the Balance Database is updated with the actual total cost of the service session. The credit information can be made accessible to Subscribers/End-users so as to provide them with (near) real-time information about their credit balance.

- Charge Record Database - The Charge Record Database stores charge records for invoicing and auditing purposes. A charge record is sent by the Charge Aggregator after the termination of a charging session.

- Tariff \& Discount Database - The Tariff \& Discount Database stores the information related to the service portfolio offered by the Service Provider. A tariff and discount plan can be tailored down to a Subscriber or End-user specific profile according to the SLA signed between the Subscriber and the Service Provider.

Figure 5 below gives an overview of the functional components comprised by the OCS and the interfaces between these functional components.

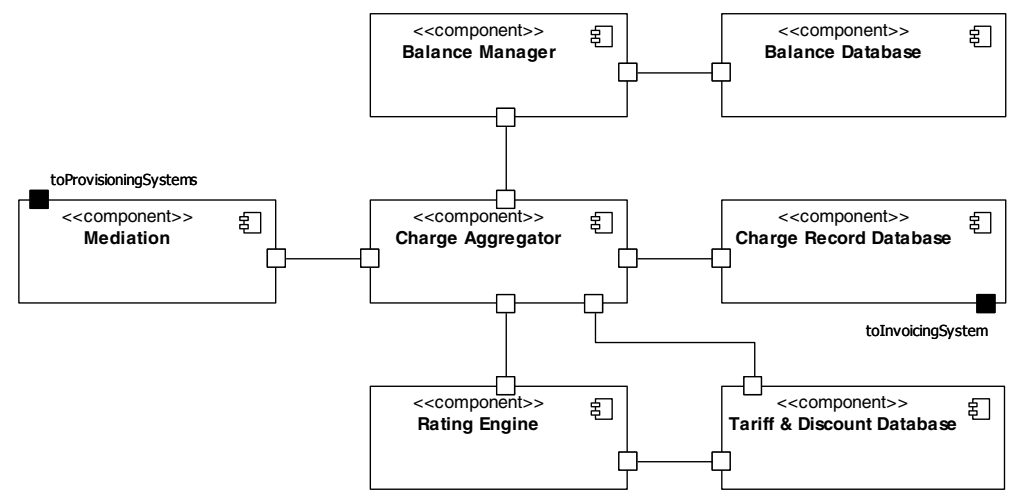

Fig. 5. Functional Components and Interfaces of the Online Charging System; the shaded ports indicates the external ports outward the OCS 


\section{Interaction between the System Components}

This section details the phases of the Online Charging System (OCS), the involved system components and their interactions at the interfaces.

\subsection{Credit Verification}

The purpose of Credit Verification is to find out whether the Subscriber's credit balance is sufficient to allow the provisioning of a requested service. When a credit verification operation arrives at the Mediation, it is forwarded to the Charge Aggregator. The Charge Aggregator looks at this request message and then tries to retrieve the estimated charge of the requested composite service session from the Tariff \& Discount Database. The reason to estimate the charge is because the duration of the requested service session is not known beforehand (e.g. the user might terminate a service session anytime). From a business perspective, it is desirable to ensure that the Subscriber has a minimum credit to be able to request a certain type of composite service. Charge estimation for different types of (composite) services can be predefined and stored in the Tariff \& Discount Database. Based on the estimated charge, the Charge Aggregator requests the Balance Manager to verify the Subscriber's credit balance. If the credit balance is sufficient, the Balance Manager returns a "positive" response. In turn, the Charge Aggregator returns a "positive" credit verification response to the Mediation, which then forwards this response to the provisioning system. If the credit balance is not sufficient, the Balance Manager returns a "negative" response. As a result, the Charge Aggregator returns a "negative" credit verification response the Mediation, which then forwards this response to the provisioning system. One of the novel aspects of the proposed design is the exchange of the service composition information in the Credit Verification case. This allows the OCS to prepare for the charging process and to estimate the charge before service provisioning. Figure 6 shows the sequential interactions between the involved functional components in the Credit Verification phase.

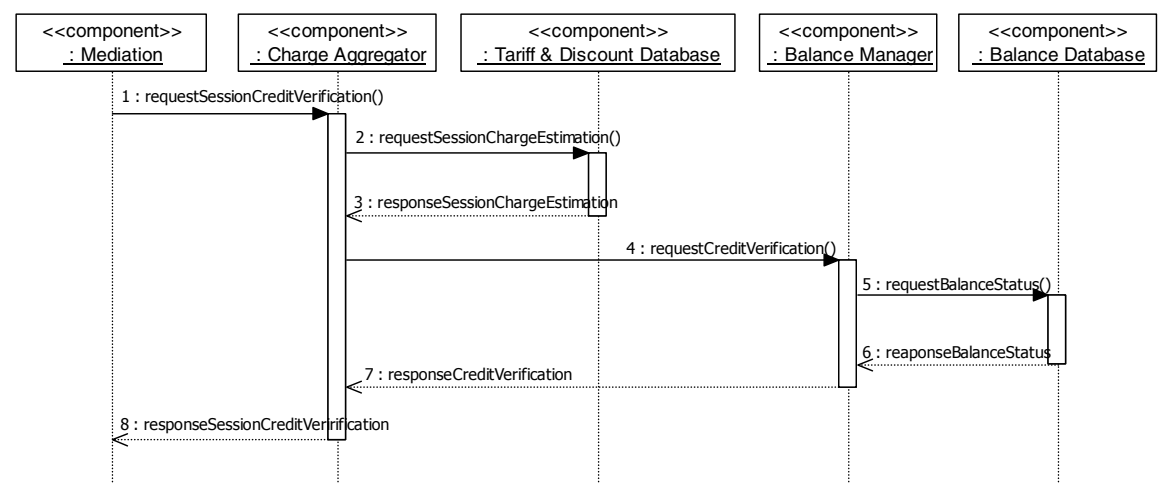

Fig. 6. Interactions between functional components involved in the Credit Verification phase 


\subsection{Accounting and Charging Instantiation}

The purpose of the Accounting and Charging Instantiation phase is to set up a charging session for individual service components of a composite service.

When a credit authorization request arrives at the Mediation, it is forwarded to the Charge Aggregator. A credit authorization request allows a (sub) provisioning system (i.e. a network element) requesting authorization to provision a service component. This operation provides the provisioning system with a possibility to submit authorization, which depends on the service unit. A service unit may be some data volume (e.g. megabyte), certain time units (e.g. second) or a number of internet pages, etc. The Service Provider may enforce provisioning sub-systems to apply a specific frequency of charge.

In order to proceed with the credit authorization request, the Charging Aggregator needs to know the cost of the service component. Therefore, it requests the Rating Engine to calculate this cost. The service component cost depends on the component tariff, the assigned discount and the service unit. Furthermore, the tariff and discount plan for a specific Subscriber and the relevant End-user may depend on a number of other parameters such as time of day, quality of service, current location of the Enduser, etc. These parameters are defined in a so-called "tariff \& discount profile" according to the SLA between the Service Provider and the Subscriber. Upon receiving the component rating request, the Rating Engine retrieves the End-user's profile from the Tariff \& Discount Database to calculate the cost.

Thereafter, the Rating Engine returns a component rating response to the Charging Aggregator, which includes the calculated cost. Note that the End-user's tariff \& discount profile is retained within the Rating Engine during the entire Charging Life Cycle. Now the cost of the service component is known, the Charge Aggregator can ask the Balance Manager to create a credit reservation by indicating the required amount. Based on the current credit balance status of the End-user, the Balance Manager creates a credit reservation and provides a response to the Charging Aggregator

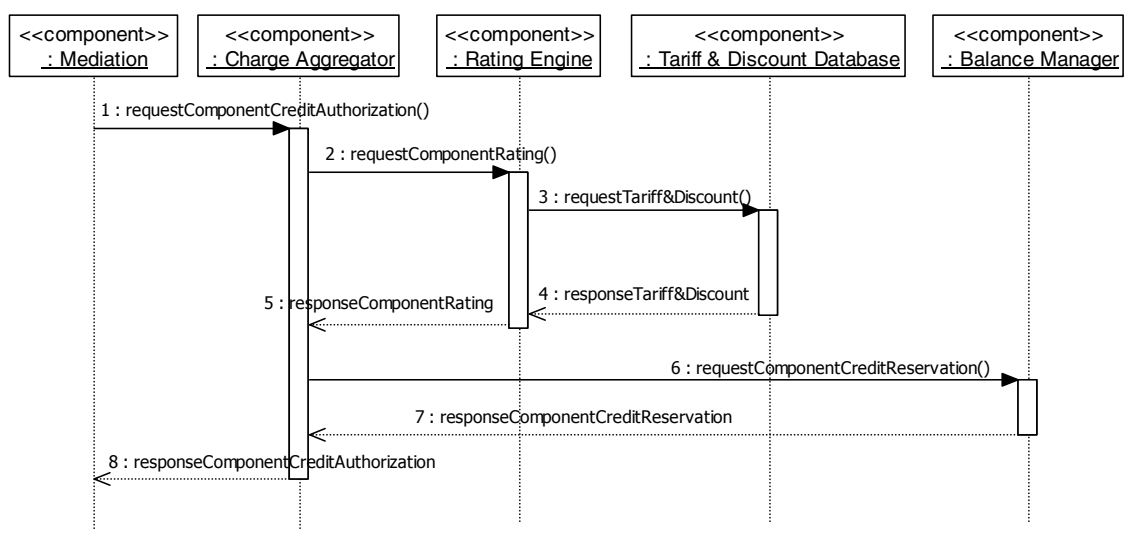

Fig. 7. Interactions between functional components involved in the Accounting and Charging Instantiation phase 
indicating that the credit reservation has been accepted. Figure 7 shows the sequential interactions between the involved functional components in the Accounting and Charging Instantiation phase.

\subsection{Accounting and Charging}

In the previous phase, service provisioning has been authorized for a certain number of service units. The purpose of the Accounting and Charging phase is to re-authorize the individual provisioning sub-systems to continue with their service provisioning. Hence, during this phase, subsequent credit re-authorization requests will be sent from the provisioning system and third party provisioning systems to the OCS.

The handing of credit-reauthorization requests arriving at the Mediation in this phase is similar to the previous phase. When a credit re-authorization request operation arrives at the Mediation, the request message is forwarded to the Charge Aggregator via the Charge Request interface. The Charge Aggregator replies to this message with a credit re-authorization response, which can be either "positive" or "negative" depending on the remaining credit balance status.

As the Charge Aggregator retains and keeps track of the costs of all continuing composite services, it can easily associate a credit re-authorization request of a specific service component with the cost, which has been calculated previously by the Rating Engine. Based on this (pre-calculated) cost, the Charge Aggregator requests the Balance Manager to create an interim credit reservation. This approach contributes to the efficiency of the Charging Aggregator and at the same time releases the load of the Rating Engine because no re-rating is necessary. Here, it is assumed that service units contained in credit re-authorization requests originating from a provisioning sub-system, remain the same. There are special occasions where the Charge Aggregator needs to ask the Rating Engine to conduct a new rating. For instance, when a credit re-authorization request arrive at the boundary of two time zones where different tariff and discounting are applied.

When the Balance Manager receives the credit re-authorization request, this request is held against the latest credit balance status so that a new credit reservation can be deducted. The Balance Manager returns a response to the Charging Aggregator, which can be either "positive" (i.e. sufficient credit balance) or "negative" (i.e. insufficient credit balance). In turn, the Charging Aggregator provides a response to

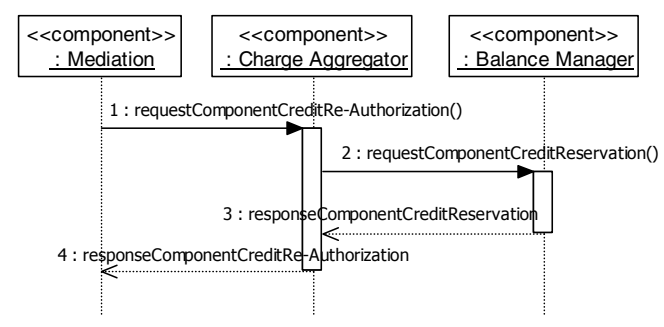

Fig. 8. Interactions between functional components involved in the Accounting and Charging phase 
the Mediation. In order to create credit reservations, the Balance Manager does not need to distinguish the difference between credit authorization and credit reauthorization. This helps to simplify its functionality and at the same time to increase its efficiency. Figure 8 shows the sequential interactions between the involved functional components in the Accounting and Charging phase.

\subsection{Accounting and Charging Termination}

In a dynamic service provisioning environment, participating service components in a composite service session might be terminated in an arbitrary order. The termination order depends on the service orchestration and service delivery logics of the provisioning system [8]. The OCS is not in control of the termination of service provisioning. Instead, it receives final usage records at the Mediation and is expected to stop the charging process for the associated service components or service session in the most logical and secure way. Hence, in this phase, it is assumed that whenever a provisioning sub-systems terminates its service provisioning, it will generate a final usage record. The final usage record contains (amongst other details) the information about the service usage of the total session, which can be either incremental or cumulative service usage.

Two termination scenarios are possible:

- The OCS first receives a service session charging termination request, and then the corresponding component charging termination requests.

- The OCS first receives different component charging termination requests, and then the corresponding service session termination request. This occurs, for example, when a service component is removed from a composite service session.

Regardless of which of the above scenarios is used, the Charging Aggregator will process every component charging termination requests that arrives. In parallel, it keeps track of all terminated service components. Once the charging processes of all service components have been terminated, the charging process of the corresponding service component can also be terminated.

When a final usage record arrives at the Mediation, the Mediation requests the Charging Aggregator to terminate the charging process of this particular service component. As the final usage may be less than the (re)-authorized service usage in the Accounting and Charging phase, the Charge Aggregator requests the Rating Engine to re-calculate the cost of the final usage. Upon this request, the Rating Engine returns a response, which contains the calculated cost of the final usage. Next, the Charging Aggregator requests the Balance Manager to release the current credit reservation of the service component in question. The Balance Manager compares the actual cost of the final usage and then it adjusts the credit reservation with this final cost. This mechanism allows the Balance Manager to manage the Subscriber's credit balance appropriately in accordance with the actual service usage. Once the credit reservation of a service component has been released, the Balance Manager returns a confirmation to the Charge Aggregator and updates the Balance Manager with the actual total cost of the service component usage.

The above termination process has a recurrent character because it repeats itself for individual service components involved in a composite service session. Once the 
charging termination of the last service component in a composite service session has been processed, the Charge Aggregator sends a notification via the Mediation to the provisioning system to report that the service session charge is (properly) terminated. Finally, the Charge Aggregator generates a charge record for the entire composite service session and stores it in the Charge Record Database. Figure 9 below shows the sequential interactions between the involved functional components in the Accounting and Charging Termination phase.

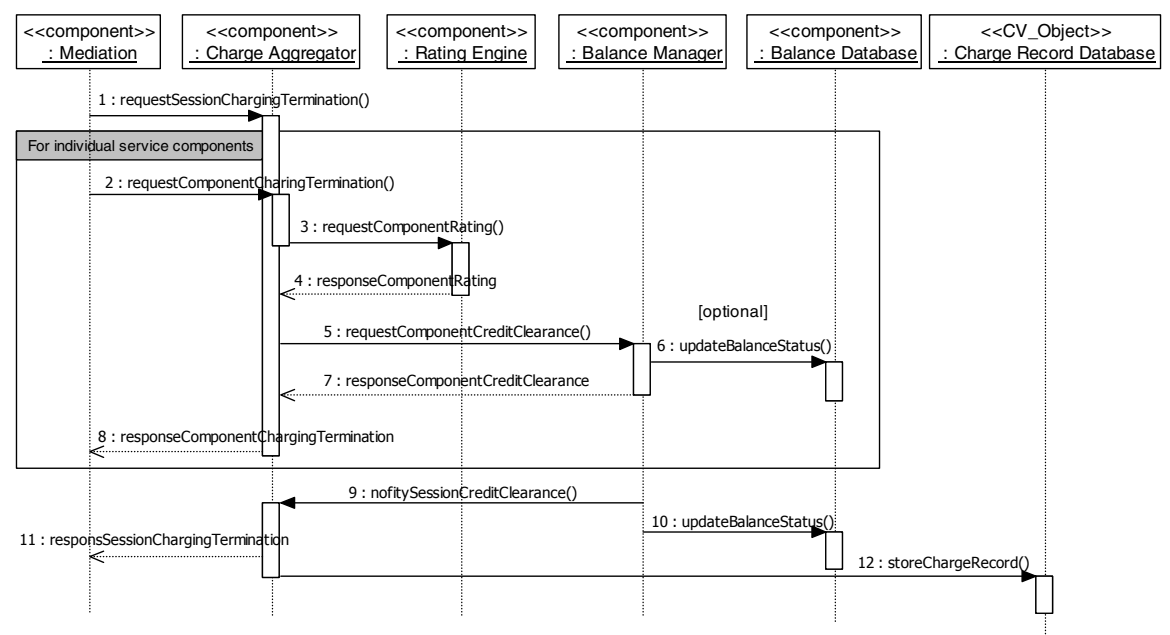

Fig. 9. Interactions between functional components involved in the Accounting and Charging Termination phase

\section{Conclusion and Future Research}

In this paper we address the problem of online charging for inter-domain composite services which are delivered across IMS-based and web-based infrastructures. A main issue here is the lacking of an adequate service composition information model that relates the constituent service components and the corresponding charging keys. In this paper we overcome this problem by giving a design of a refined IMS based Online Charging System (OCS) together with a composition model based on the SID framework of the TM Forum. Since the design applies the principle of separation of concerns one may expect that it simplifies an implementation and support a large variety of business models between service partners in different domains.

Future investigation on performance aspects of the system components embodies by the proposed OCS will provide insights about their performance indicators. In particular, attention will be paid to the charge aggregation system component since this component is expected to be computing intensive. To achieve high performance, efficient engineering techniques will be explored in combination with state-of-the-art technology such as in-memory databases. 


\section{References}

1. Cuevas, A., Moreno, J.I., Vidales, P., Einsiedler, H.: The IMS service platform: a solution for next-generation network operators to be more than bit pipes. IEEE Communication Magazine 44(8) (August 2006)

2. 3GPP, Charging Architecture and Principles, TS 32.240, version 8.4.0 (September 2008)

3. Jahnert, J., Wesner, S.: Overall Architecture Definition and Layer Integration, Akogrimo, deliverable 3.1.1 (July 2005)

4. de Laat, C., Gross, G., Gommans, L., Vollbrecht, J., Spence, D.: Generic AAA Architecture, IETF RFC 2903 (August 2000)

5. Koutsopoulou, M., et al.: Charging, Billing \& Accounting in a multi-Operator and multiService Provider Environment. In: ANWIRE, Workshop on Reconfigurability, Mykonos Greece (September 2003)

6. Huslak, N.S., McQuaide, A.C.: Service Brokering: Opportunities and Challenges. In: International Conference on Intelligent Network (ICIN), Bordeaux France (October 20-23, 2008)

7. 3GPP, Study on Architecture Impacts of Service Brokering, TR 23.810, release 8.0 (September 2008)

8. TMForum, Service Delivery Framework Overview, release 2.0 (September 2008)

9. TMForum, Shared Information/Data (SID) Model - Business View Concepts, Principles and Domains, release 6 (November 2005)

10. van Le, M., Beijnum, B.J.F., Nieuwenhuis, L.J.M., Huitema, G.B.: An Enterprise Model for Real-time Inter-domain Billing of Services. In: Workshop on ODP Enterprise Computing (WODPEC), Muchen, Germany (September 2008)

11. van Le, M., Rumph, F.J., Huitema, G.B., Beijnum, B.J.F., Nieuwenhuis, L.J.M.: Online Charging for IMS-based Inter-Domain Composite Services. In: 6th International Workshop on Internet Charging and QoS Technologies, Aachen (May 2009)

12. van Le, M., van Beijnum, B.J.F., de Goede, B.L.: Real-time Service Accounting. In: IEEE Workshop on IP Operations and Management (IPOM2002), Dallas, TX, USA, October 29-31 (2002)

13. Xu, L., Jennings, B.: Automating the Generation, Deployment and Application of Charging Schemes for Composed IMS Services. In: IFIP/IEEE International Symposium on Integrated Network Management (IM 2007), Munich, Germany, May 21-25 (2007) 